\title{
Computed tomographic evaluation of bone tunnel enlargement following single bundle anterior cruciate ligament reconstruction with hamstring autograft
}

\author{
Sunil Chandrashekar*, Manjunath A. N., Shivakumar M. S.
}

Department of Orthopaedics, East Point College of Medical Sciences, Bengaluru, Karnataka, India

Received: 04 October 2021

Revised: 22 November 2021

Accepted: 30 November 2021

\section{*Correspondence:}

Dr. Sunil Chandrashekar,

E-mail: drsunilctumkur@gmail.com

Copyright: (c) the author(s), publisher and licensee Medip Academy. This is an open-access article distributed under the terms of the Creative Commons Attribution Non-Commercial License, which permits unrestricted non-commercial use, distribution, and reproduction in any medium, provided the original work is properly cited.

\begin{abstract}
The purpose of this study is to evaluate tibial and femoral tunnel diameter following single bundle anterior cruciate ligament (ACL) reconstruction and correlation between tunnel enlargement and clinical outcome. Twelve patients who underwent primary arthroscopic single bundle ACL reconstruction with hamstring graft were included in prospective case series. Preoperative clinical evaluation was performed using international knee documentation committee (IKDC) subjective score and grade, Tegner knee score and Lysholm knee score. Computed tomography (CT) evaluation of the femoral and tibial tunnels were done on post-operative day (POD) 1 and at mean follow up of 9 months (range 7-12 months) and were compared with functional scores. Our study shows significant tibial and femoral tunnel enlargement on CT scan at 9 months (range 7-12 months) postoperatively. All the clinical evaluation scales showed improvement postoperatively. The mean average femoral tunnel diameter increased significantly $(\mathrm{p}<0.001)$ from $8.17 \pm 0.57$ to $9.08 \pm 0.660(10 \%)$ and tibial tunnel diameter increased significantly $(\mathrm{p}<0.001)$ from $8.08 \pm 0.669$ to $9.07 \pm 0.601(11 \%)$ postoperatively at a mean follow up of 9 months (range 7-12 months). No statistically significant difference between tunnel enlargement and clinical values were found. In our current prospective CT based study, we conclude use of extracortical fixation of femoral tunnel with stronger fixation of the tibial tunnel, tunnel orientation and anatomic fixation close to the joint line along with less aggressive rehabilitation protocol with use of extension knee brace may result in minimization of tunnel widening with quadrupled hamstring autograft.
\end{abstract}

Keywords: Anterior cruciate ligament, Tunnel widening, Hamstring autograft, CT, Tibial tunnel, Femoral tunnel

\section{INTRODUCTION}

Anterior cruciate ligament (ACL) reconstruction is one of the commonly performed surgeries all over the world. The enlargement of tibial and femoral bone tunnels is commonly encountered postoperatively. ${ }^{1-7}$ Computed tomography (CT) scan remains gold standard to X-ray and magnetic resonance imaging (MRI) in measuring the cross-sectional area of bone tunnels. ${ }^{8,9}$ The enlargement of tunnels can occur anywhere along its cross sectional area. The reported incidence of tunnel widening (TW) inconsistently varies among different studies, ranging from 29 to $100 \%$ for tibia and 25 to $100 \%$ for femur. ${ }^{10}$ Etiopathogenesis of TW is still obscure and thought to be multifactorial, a complex interaction between mechanical and biological factors exists. ${ }^{11}$ Amongst possible biological cause include immune response to foreign material (allograft), cell necrosis due to heat generated from drill bit and toxic products released from it, also due to nonspecific immune mediated release of cytokines and other inflammatory cells from the synovial fluid which seeps between the graft and the bone. ${ }^{11}$ Mechanical factors like graft type, fixation method, excess graft motion within the tunnel, improper tunnel placement, original bone 
diameter, inappropriate surgical planning, aggressive rehabilitation may also contribute. ${ }^{11-14}$ It has also been reported that farther the fixation points on the tibia or the femur more is the widening of the tunnel. Hence fixation type along with the tunnel placement should be focused on as these are surgeon dependent. ${ }^{4,15}$

Tunnel widening is important for the following reasons: it is important to preserve the bone stock in revision ACL surgeries; and it acts as a stress riser resulting in bone fracture. ${ }^{16}$ Male sex, younger age and delayed ACL reconstruction ( $>1$ year from the time of injury) is associated with significant $\mathrm{TW} .{ }^{17}$

The purpose of this study is to prospectively evaluate the TW in the femur and tibia following single bundle ACL reconstruction with hamstring autograft using anteromedial portal technique and to evaluate the association between TW and clinical scores.

\section{CASE SERIES}

This is a prospective observational study conducted at a tertiary level teaching hospital from May 2019 to September 2019 with an average follow up of 9 months (range 8-12 months). Our study included twelve patients of whom 11 are male and 1 female with a mean age of $28.25 \pm 7.875$.

Inclusion criteria were complete ACL tear with or without meniscal injury with normal contralateral knee.

Exclusion criteria were multiligamentous injury, instability due to malunited fractures, outerbridge grade 3 or grade 4 arthritic changes or neuromuscular diseases.

All underwent arthroscopic ACL reconstruction using hamstring autograft which is quadrupled. Ten patients underwent anteromedial portal technique and the reminder underwent all inside technique. All femoral fixation was done using endobutton and tibial fixation with biointerference screw and endobutton.

Intraoperative femoral tunnel diameters of 8 patients were $8 \mathrm{~mm}, 3$ patients had $9 \mathrm{~mm}$ and 1 patient had $7 \mathrm{~mm}$ with tibial tunnel of 7 patients were $8 \mathrm{~mm}, 3$ patients had $9 \mathrm{~mm}$ and 2 patients with $7 \mathrm{~mm}$ diameters. The graft tunnel mismatch were $<1 \mathrm{~mm}$ as calculated by postoperative CT.

Preoperative clinical evaluation of knee function and stability was done using international knee documentation committee (IKDC) 2000 subjective knee evaluation score and IKDC grade, Lysholm knee score and Tegner activity level.

\section{Postoperative treatment and rehabilitation}

Postoperatively, every patient's knee was placed in long extension knee brace with ice packs for control of pain and inflammation. From the $2^{\text {nd }}$ postoperative day (POD) isometric quadriceps with ankle pumping exercises were started. Patients were allowed to walk with partial weight bearing for the first two weeks and full weight bearing thereafter with walker. Closed chain range-of-motion (ROM) exercises were started from POD-2 to reach $90^{\circ}$ by the end of 2 weeks and full ROM by the end of 4 weeks. Knee brace and walker were discarded at the end of 4 weeks. Muscle strengthening and open chain ROM exercises were started at the end of 6 weeks. At the $3^{\text {rd }}$ month onwards isotonic, isokinetic exercises and progressive functional activities (cycling, running) are started. Complete return to activity by $6-9$ months.

\section{Radiographic evaluation}

CT of the femoral and tibial tunnels were evaluated at post op day 1 and during follow up period. The examinations were performed using Siemens 32 slice multislice CT (MSCT) with post-processing multislab reconstruction on the coronal, axial and sagittal planes. Bone tunnel measurements were made perpendicular to the long axis of the tunnel. MSCT scanning was performed at a level just above the outer femoral tunnel to a level below the outer hole of the tibial tunnel. The slice thickness is $1 \mathrm{~mm}$, with retro-reconstruction of $0.5 \mathrm{~mm}$ made in all patients.

We measured the transosseous tibial and femoral tunnel diameters at 8 different levels, four each for tibial and femoral tunnels.

The reference tunnel diameters for tibial and femoral tunnels are taken as follows: F1 femoral tunnel axial at notch, F2 femoral tunnel axial at middle point, F3 femoral tunnel coronal at middle point, F4 femoral tunnel sagittal at middle point, T1 tibial tunnel, axial at plateau, T2 tibial tunnel, axial at middle point, T3 tibial tunnel, coronal at middle point, and T4 tibial tunnel, sagittal at middle point (Figures 1-4).

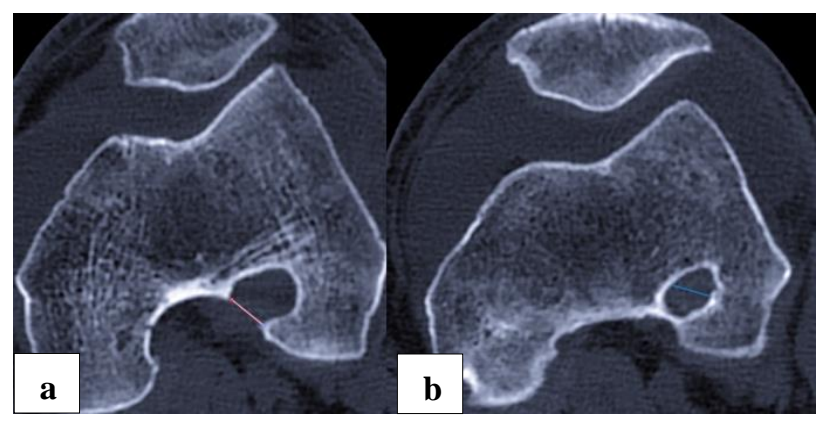

Figure 1: Tibial and femoral tunnels 1.

Tunnel diameters were calculated in millimeters.

\section{Postoperative follow-up}

Twelve patients were reviewed after a mean period 9 months (range 7-12) with clinical assessment using IKDC 2000 subjective knee evaluation score, IKDC grade, Tegner activity level and Lysholm knee score. 
Repeat CT scanning were performed using the same criteria described in the immediate postoperative evaluation.

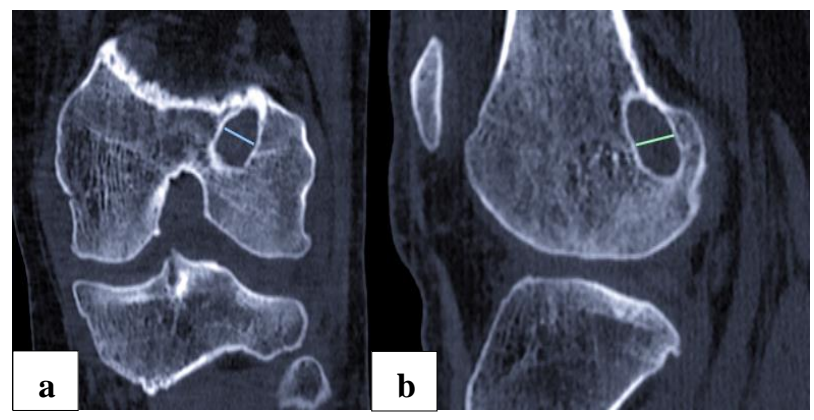

Figure 2: Tibial and femoral tunnels 2.

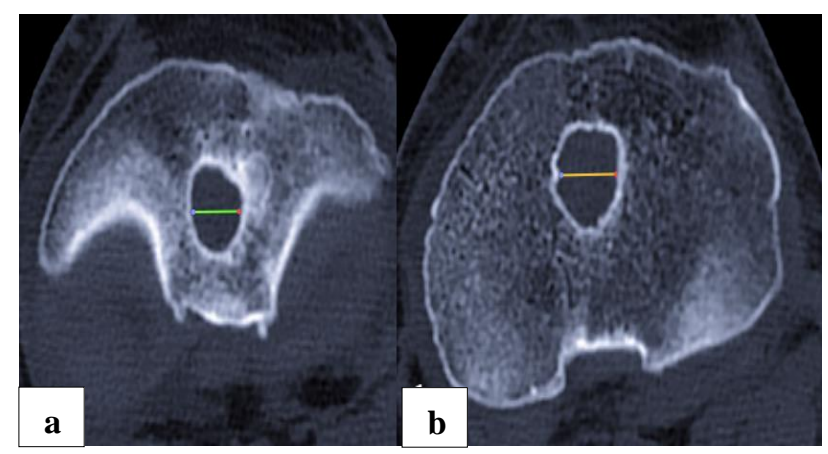

Figure 3: Tibial and femoral tunnels 3.

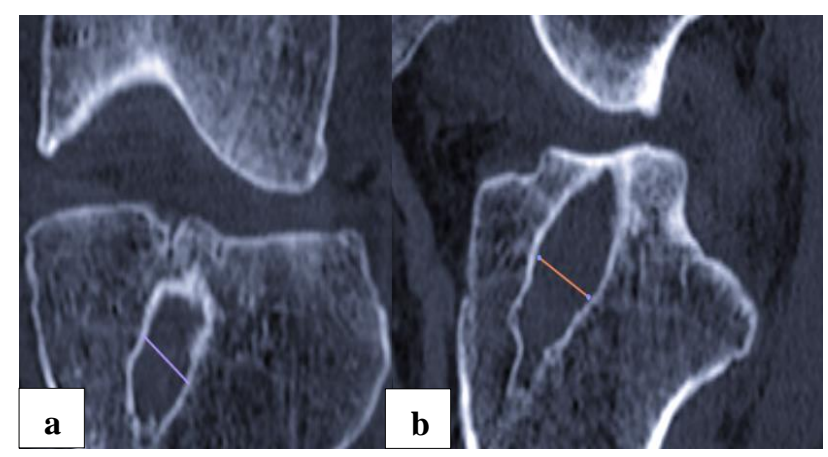

Figure 4: Tibial and femoral tunnels 5.

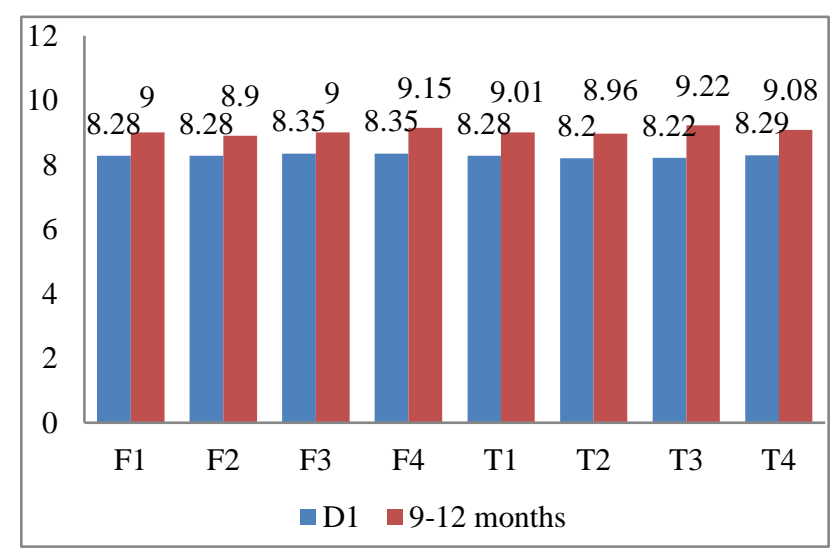

Figure 5: Tunnel diameters.

\section{Statistical analysis}

Based on the previous publication by Iorio et al, the mean \pm SD of tibial tunnel diameter values were $(9.03 \pm 0.04$ $\mathrm{mm}, 10 \pm 0.8 \mathrm{~mm}$ ) with $95 \%$ confidence level and $80 \%$ statistical power, the minimum sample size was calculated to be $12 .^{12}$

For calculation of statistical significance of continuous variables Student's t-test was used and for categorical variables analysis of variance (ANOVA) test was used. $\mathrm{P}$ value $<0.05$ was taken as statistically significant. Of the 12 patients included in our study, 11 are male 1 female with mean age of $28.25 \pm 7.875$. 5 patients had right sided tear and 7 patients had left sided tear. ACL reconstruction with medial meniscus repair done in 2 patients, with medial meniscal debridement done in 1 patient and rest of them underwent isolated single bundle ACL reconstruction. None of the patients had complications like deep vein thrombosis, nerve injuries or infection. Full range of motion was seen in all patients.

The mean IKDC 2000 subjective score increased from $45.92 \pm 10.604$ preoperatively to $78.00 \pm 4.843$ post operatively. The mean Lysholm knee score increased from $70.17 \pm 5.323$ preoperatively to $90.33 \pm 5.033$ postoperatively. The mean Tegner activity score improved from $3.25 \pm 0.754$ preoperatively to $6.58 \pm 0.515$ postoperatively. On the IKDC objective grade 8 patients (66\%) were grded C and 4 patients $(33 \%)$ were graded D preoperatively. Postoperatively 5 patients $(41 \%)$ were graded A, and 7 patients (58\%) were graded B (Table 1).

\section{Femoral tunnels}

CT scan obtained on POD 1 showed mean average increase in the femoral tunnel diameter from $8.17 \pm 0.57$ to $9.08 \pm 0.660$ during the follow up period with a $p$ value $<0.001$ which is significant (Table 2).

Comparison of tunnel diameter of femur during the follow up period to the IKDC objective grade showed no statistical difference with a p value 0.408 (Table 3 ).

Comparison of tunnel diameter of femur during the follow up period to the Lysholm knee score showed no statistical difference with a $\mathrm{p}$ value 0.618 (Table 3 ).

\section{Tibial tunnels}

CT scan obtained on POD 1 showed mean average increase in the tibial tunnel diameter from $8.08 \pm 0.669$ to $9.07 \pm 0.601$ during the follow up period with a $\mathrm{p}$ value $<0.001$ which is significant (Table 2). Comparison of tunnel diameter of tibia during the follow up period to the IKDC objective grade showed no statistical difference with a $\mathrm{p}$ value 0.876 (Table 3). Comparison of tunnel diameter of tibia during the follow up period to the Lysholm knee score showed no statistical difference with a p value 0.552 (Table 3 ). 
Table 1: Preoperative and follow up clinical scores (IKDC) $(\mathrm{n}=12)$.

\begin{tabular}{|ll|l|}
\hline Parameters & $\begin{array}{l}\text { Pre-op } \\
(\text { mean } \pm \text { SD })\end{array}$ & $\begin{array}{l}\text { Follow up } \\
(\text { mean } \pm \text { SD })\end{array}$ \\
\hline $\begin{array}{l}\text { IKDC subjective } \\
\text { score }\end{array}$ & $45.92 \pm 10.604$ & $78.00 \pm 4.843$ \\
\hline IKDC grade & $\begin{array}{l}\mathrm{A}=0, \mathrm{~B}=0, \mathrm{C}=8, \\
\mathrm{D}=4\end{array}$ & $\begin{array}{l}\mathrm{A}=5, \mathrm{~B}=7, \\
\mathrm{C}=0, \mathrm{D}=0\end{array}$ \\
\hline $\begin{array}{l}\text { Lysholm knee } \\
\text { score }\end{array}$ & $70.17 \pm 5.323$ & $90.33 \pm 5.033$ \\
\hline Tegner score & $3.25 \pm 0.754$ & $6.58 \pm 0.515$ \\
\hline
\end{tabular}

Table 2: Comparison of day 1 FT and TT and follow up FT and TT values.

\begin{tabular}{|llll|}
\hline Variable & $\begin{array}{l}\text { D1 scores } \\
(\text { mean } \pm \text { SD })\end{array}$ & $\begin{array}{l}\text { F/U score } \\
(\text { mean } \pm \text { SD })\end{array}$ & $\begin{array}{l}\text { P } \\
\text { value }\end{array}$ \\
\hline $\begin{array}{l}\text { Femoral } \\
\text { tunnel (FT) }\end{array}$ & $8.17 \pm 0.57$ & $9.08 \pm 0.660$ & $<0.001$ \\
\hline $\begin{array}{l}\text { Tibial tunnel } \\
\text { (TT) }\end{array}$ & $8.08 \pm 0.669$ & $9.07 \pm 0.601$ & $<0.001$ \\
\hline
\end{tabular}

Table 3: Comparative analysis of clinical values with follow up femoral and tibial tunnel diameters $(n=12)$.

\begin{tabular}{|c|c|c|c|}
\hline Parameters & $\begin{array}{l}\text { Clinical } \\
\text { assessment }\end{array}$ & $\begin{array}{l}\text { Tunnel } \\
\text { diameter }\end{array}$ & $\begin{array}{l}P \\
\text { value }\end{array}$ \\
\hline \multirow{2}{*}{ FT } & IKDC grade A & $9.40 \pm 0.548$ & \multirow{2}{*}{0.408} \\
\hline & IKDC grade B & $9.00 \pm 0.577$ & \\
\hline \multirow{2}{*}{ TT } & IKDC grade A & $9.27 \pm 0.56$ & \multirow{2}{*}{0.876} \\
\hline & IKDC grade $\mathrm{B}$ & $8.92 \pm 0.62$ & \\
\hline \multirow{3}{*}{$\begin{array}{l}\text { FT - LKS } \\
\text { post op } \\
\text { score }\end{array}$} & $>90(\mathrm{n}=6)$ & $9.20 \pm 0.534$ & \multirow{3}{*}{0.618} \\
\hline & $84-90(n=4)$ & $8.93 \pm 0.239$ & \\
\hline & $65-83(n=2)$ & $8.75 \pm 1.41$ & \\
\hline \multirow{3}{*}{$\begin{array}{l}\text { TT - LKS } \\
\text { post op } \\
\text { score }\end{array}$} & $>90(\mathrm{n}=6)$ & $8.83 \pm 0.672$ & \multirow{3}{*}{0.552} \\
\hline & $84-90(n=4)$ & $9.24 \pm 0.427$ & \\
\hline & $65-83(n=2)$ & $9.42 \pm 0.671$ & \\
\hline
\end{tabular}

LKS- Lysholm knee score.

\section{DISCUSSION}

Femoral and tibial TW after single or double ACL reconstruction is a well proven fact documented in most of the literature. It is important factor for consideration during revision ACL surgeries as it requires the need for bone grafting of the enlarged tunnels as a single or 2 stage procedure. ${ }^{18}$ Previous studies indicate there is no correlation between tunnel enlargement and clinical scores by using digital calipers for the measurement of tunnel width in both anteroposterior and lateral radiographs for a follow up of 2 years. ${ }^{1,7}$ One study by Webster et al found $24 \%$ to $38 \%$ error in measuring the tunnel width on X-ray and advised caution while measuring small changes. ${ }^{19} \mathrm{X}$ ray measurement results in underestimation of tunnel size due to improper magnification and the presence of interference screw within the bone tunnels. In our study we used MSCT images for accurate depiction of transosseous femoral and tibial tunnels. CT is considered gold standard in evaluation of trans osseous tunnels compared to $\mathrm{X}$ rays and MRI. ${ }^{8,9}$ Geometric factors such as position change of knee, distance between the source and the film surface are not affected by the $\mathrm{CT}$ scan when compared to $\mathrm{X}$ rays. POD 1 CT measurements closely resembled that of drill bit size used intraoperatively.

Tunnel widening following ACL reconstruction depends on the method of fixation used, type of graft used and position of fixation site. In a prospectively randomized CT based study by Sabat et al compared transfix and endobutton group with TW and found TW is significant in endobutton group compared to transfix during 1 year follow up period and TW was decreased at the aperture on the tibial side when the screw tip was 10 to $15 \mathrm{~mm}$ away from the aperture. ${ }^{4}$ Fauno et al reported significant reduction in TW in both femur and tibia when fixation points close to the joint. ${ }^{15}$ Peyrache et al reported an increase in tunnel diameter at 3 months, no significant change from 3 months to 2 years and decrease at 3 years after surgery. ${ }^{20}$ Tunnel widening begins in the immediate postoperative period which increases significantly in the first 6 weeks and gradually progresses thereafter for a period of 2-3 years and then ceases. ${ }^{21,22}$ Recently Shimizu et al found tunnel enlargement starts as early as 2 weeks post ACL reconstruction. ${ }^{23}$

In accordance with the above authors tunnel widening is maximum during the $1^{\text {st }}$ year and our investigation of tunnel widening during average follow up of 9 months will be ideal.

Jansson et al noted an average increase in femoral tunnel diameter by $33 \%$ and tibial tunnel diameter by $23 \%$ detected on anteroposterior radiograph during 2 year follow up period. ${ }^{34}$ Reconstruction was done using hamstring autograft (HA) and endobutton fixation. L'Insalata et al observed mean percentage increase in the tunnel size after ACL reconstruction with hamstring (HS) graft on posteroanterior view (PA); it was $20.9 \pm 13.4 \%$ for tibial tunnel and $30.2 \pm 17.2 \%$ for femoral tunnel. ${ }^{3}$ In the lateral view it was $25.5 \pm 16.7 \%$ for tibial tunnel and $28.1 \pm 14.7 \%$ for femoral tunnel for a mean follow up period of 9 months. Fules et al observed tibial tunnel cross sectional area enlargement by $33 \%$ after ACL reconstruction with $\mathrm{HS}$ graft on MRI at a mean follow up of 6.5 months. $^{24}$

Our study results demonstrate tunnel enlargement of $11 \%$ for tibia and $10 \%$ for femur following ACL reconstruction with HA, which is much lower compared to the studies described above. TW is a major concern during revision ACL surgery and reconstruction of multiligamentous injuries. There has been a case report of tibial plateau fracture after ACL reconstruction due to marked osteolysis of bone around the interference screw which acts as a stress riser. ${ }^{16}$

Few studies compared bone tunnel enlargement between bone patellar tendon bone (BPTB) graft and HA. One 
study by L'Insalata et al has shown greater enlargement of bone tunnels among HS compared to BPTB; point of fixation of HA are at a greater distance from the normal anatomic footprint compared to BPTB graft which creates a large amount of force while graft cycling leading to expansion of tunnel (windshield-wiper effect). ${ }^{3}$ Three studies have shown there is greater increase in the femoral tunnel in HS group compared to BPTB graft. Hersekli et al found tibial tunnel enlargement were similar in both HA and BPTB graft while femoral tunnel was significantly larger in HA. ${ }^{25}$ Webster et al suggested biological healing of bone to bone compared to tendon to bone healing of HA reduces tunnel widening among BPTB graft. ${ }^{7}$ However Clatworthy et al considered tunnel widening as more than $50 \%$ increase in the width of femoral and tibial tunnels. ${ }^{2}$ Marked enlargement of tunnels were found in HA compared to subtle enlargement in BPTB graft probably due to marked variation in the placement of tunnels in both groups.

The aetiology of TW following ACL reconstruction is multifactorial and no one cause has been determined so far. Several potential mechanisms have been put forward by various authors. Some include biological factors such as foreign body reaction to allograft, nonspecific response to inflammatory cells, heat necrosis of bone arising from drilling, and release of cytokines such as IL-6, IL-8 and tumour necrosis factor (TNF- $\alpha$ ) at the time of graft necrosis leading to osteolysis of bone..$^{2,7,26,27}$ Mechanical factors include improper tunnel placement, excessive motion of graft within the tunnel, stress deprivation of bone within tunnel and aggressive rehabilitation. ${ }^{28,29} 2$ types of motion of graft occurs within the tunnel: longitudinal motion of graft within the tunnel (bungee cord effect) and transverse motion of graft within the tunnel (windshield wiper effect). ${ }^{30}$

In our study we used extracortical fixation (endobutton) for femur and aperture fixation (bio interference screw) or endobutton for tibia. The fixation devices are strong, stiff and with higher load to failure, anatomical fixation of graft close to the joint line could contribute to lesser incidence of tunnel enlargement.

Silva et al conducted MRI evaluation of femoral tunnel at 3 months follow up following ACL reconstruction with $\mathrm{HA}$ and concluded biological factors contributed more to tunnel widening than mechanical factors. ${ }^{6}$ Some authors investigated relationship between tunnel widening and rehabilitation, in a review article by Bhullar et al, it took an average of around 9.5 months to diagnose tunnel osteolysis following ACL reconstruction on CT scan, double bundle HA demonstrated lower tibial and femoral TW than the single bundle HA and extension locked bracing following ACL reconstruction had the lowest TW of all the bracing protocols. ${ }^{31}$

Murty et al suggested 2 weeks of immobilisation resulted in increased TW. ${ }^{5}$ Non aggressive rehabilitation not only improves the functional recovery but also better healing of the graft. $^{29}$ Increase in the osteolytic cytokines in the synovial fluid which percolates through the gap between the graft and the bone resulting in widening of the tunnel. ${ }^{2,27}$

Our study followed less aggressive rehabilitation protocol, more secure fixation of the graft with endobutton and bio interference screw and more anatomic fixation using anteromedial portal technique close to the joint line which might have resulted in decrease in TW during a follow up period of 9 months (range 7-12).

We compared tibial and femoral TW with the IKDC grade and lysholm knee scores and found no statistically significant difference between them as per other studies. ${ }^{1,2,21,32,33}$

Several limitations are present in our current study, short follow up and sample size. Larger sample size with randomization and multicentric studies are needed. Further studies with longer follow up are needed to establish the role of different fixation techniques, graft used for reconstruction and rehabilitation on the magnitude of TW in both femur and tibia. The exact etiopathogenesis of TW is still a mystery and multiple factors are to be considered in this regard.

\section{CONCLUSION}

In our prospective observational, clinical and CT based study; the rate of tunnel widening appears to be lower compared to previous studies. There is no statistically significant difference in clinical scores with tunnel widening. We conclude use of extracortical fixation of femoral tunnel with stronger fixation of the tibial tunnel, tunnel orientation and anatomic fixation close to the joint line along with less aggressive rehabilitation protocol with use of extension knee brace may result in minimization of TW with quadrupled HA.

\section{Funding: No funding sources Conflict of interest: None declared Ethical approval: Not required}

\section{REFERENCES}

1. Buelow J-U, Siebold R, Ellermann A. A prospective evaluation of tunnel enlargement in anterior cruciate ligament reconstruction with hamstrings: extracortical versus anatomical fixation. Knee Surg Sports Traumatol Arthrosc Off J ESSKA. 2002;10(2):80-5.

2. Clatworthy MG, Annear P, Bulow JU, Bartlett RJ. Tunnel widening in anterior cruciate ligament reconstruction: a prospective evaluation of hamstring and patella tendon grafts. Knee Surg Sports Traumatol Arthrosc Off J ESSKA. 1999;7(3):138-45.

3. L'Insalata JC, Klatt B, Fu FH, Harner CD. Tunnel expansion following anterior cruciate ligament reconstruction: a comparison of hamstring and 
patellar tendon autografts. Knee Surg Sports Traumatol Arthrosc Off J ESSKA. 1997;5(4):234-8.

4. Sabat D, Kundu K, Arora S, Kumar V. Tunnel widening after anterior cruciate ligament reconstruction: a prospective randomized computed tomography--based study comparing 2 different femoral fixation methods for hamstring graft. Arthrosc J Arthrosc Relat Surg Off Publ Arthrosc Assoc N Am Int Arthrosc Assoc. 2011;27(6):776-83.

5. Murty AN, el Zebdeh MY, Ireland J. Tibial tunnel enlargement following anterior cruciate reconstruction: does post-operative immobilisation make a difference? The Knee. 2001;8(1):39-43.

6. Silva A, Sampaio R, Pinto E. Femoral tunnel enlargement after anatomic ACL reconstruction: a biological problem? Knee Surg Sports Traumatol Arthrosc Off J ESSKA. 2010;18(9):1189-94.

7. Webster KE, Feller JA, Hameister KA. Bone tunnel enlargement following anterior cruciate ligament reconstruction: a randomised comparison of hamstring and patellar tendon grafts with 2-year follow-up. Knee Surg Sports Traumatol Arthrosc Off J ESSKA. 2001;9(2):86-91.

8. Marchant MH, Willimon SC, Vinson E, Pietrobon R, Garrett WE, Higgins LD. Comparison of plain radiography, computed tomography, and magnetic resonance imaging in the evaluation of bone tunnel widening after anterior cruciate ligament reconstruction. Knee Surg Sports Traumatol Arthrosc Off J ESSKA. 2010;18(8):1059-64.

9. de Beus A, Koch JE, Hirschmann A, Hirschmann MT. How to evaluate bone tunnel widening after ACL reconstruction - a critical review. Muscles Ligaments Tendons J. 2017;7(2):230-9.

10. Stolarz M, Ficek K, Binkowski M, Wróbel Z. Bone tunnel enlargement following hamstring anterior cruciate ligament reconstruction: a comprehensive review. Phys Sportsmed. 2017;45(1):31-40.

11. Taketomi S, Inui H, Sanada T, Yamagami R, Tanaka S, Nakagawa T. Eccentric Femoral Tunnel Widening in Anatomic Anterior Cruciate Ligament Reconstruction. Arthrosc J Arthrosc Relat Surg. 2014;30(6):701-9.

12. Iorio R, Vadalà A, Argento G, Di Sanzo V, Ferretti A. Bone tunnel enlargement after ACL reconstruction using autologous hamstring tendons: a CT study. Int Orthop. 2007;31(1):49-55.

13. Sauer S, Lind M. Bone Tunnel Enlargement after ACL Reconstruction with Hamstring Autograft Is Dependent on Original Bone Tunnel Diameter. Surg J N Y N. 2017;3(2):96-100.

14. Samitier G, Marcano AI, Alentorn-Geli E, Cugat R, Farmer KW, Moser MW. Failure of Anterior Cruciate Ligament Reconstruction. Arch Bone Jt Surg. 2015;3(4):220-40.

15. Fauno P, Kaalund S. Tunnel widening after hamstring anterior cruciate ligament reconstruction is influenced by the type of graft fixation used: a prospective randomized study. Arthrosc J Arthrosc
Relat Surg Off Publ Arthrosc Assoc N Am Int Arthrosc Assoc. 2005;21(11):1337-41.

16. Thaunat M, Nourissat G, Gaudin P, Beaufils P. Tibial plateau fracture after anterior cruciate ligament reconstruction: Role of the interference screw resorption in the stress riser effect. The Knee. 2006;13(3):241-3.

17. Weber AE, Delos D, Oltean HN, Vadasdi K, Cavanaugh J, Potter HG, et al. Tibial and Femoral Tunnel Changes After ACL Reconstruction: A Prospective 2-Year Longitudinal MRI Study. Am J Sports Med. 2015;43(5):1147-56.

18. Weiler A, Richter M, Schmidmaier G, Kandziora F, Südkamp NP. The EndoPearl device increases fixation strength and eliminates construct slippage of hamstring tendon grafts with interference screw fixation. Arthrosc J Arthrosc Relat Surg Off Publ Arthrosc Assoc N Am Int Arthrosc Assoc. 2001;17(4):353-9.

19. Webster KE, Chiu JJ, Feller JA. Impact of measurement error in the analysis of bone tunnel enlargement after anterior cruciate ligament reconstruction. Am J Sports Med. 2005;33(11):1680-7.

20. Peyrache MD, Djian P, Christel P, Witvoet J. Tibial tunnel enlargement after anterior cruciate ligament reconstruction by autogenous bone-patellar tendonbone graft. Knee Surg Sports Traumatol Arthrosc Off J ESSKA. 1996;4(1):2-8.

21. Fink C, Zapp M, Benedetto KP, Hackl W, Hoser C, Rieger M. Tibial tunnel enlargement following anterior cruciate ligament reconstruction with patellar tendon autograft. Arthrosc J Arthrosc Relat Surg Off Publ Arthrosc Assoc N Am Int Arthrosc Assoc. 2001;17(2):138-43.

22. Harris NL, Indelicato PA, Bloomberg MS, Meister $\mathrm{K}$, Wheeler DL. Radiographic and Histologic Analysis of the Tibial Tunnel after Allograft Anterior Cruciate Ligament Reconstruction in Goats. Am J Sports Med. 2002;30(3):368-73.

23. Shimizu R, Adachi N, Ishifuro $M$, Nakamae A, Ishikawa $\mathrm{M}$, Deie $\mathrm{M}$, et al. Bone tunnel change develops within two weeks of double-bundle anterior cruciate ligament reconstruction using hamstring autograft: A comparison of different postoperative immobilization periods using computed tomography. The Knee. 2017;24.

24. Fules PJ, Madhav RT, Goddard RK, NewmanSanders A, Mowbray MAS. Evaluation of tibial bone tunnel enlargement using MRI scan cross-sectional area measurement after autologous hamstring tendon ACL replacement. The Knee. 2003;10(1):87-91.

25. Hersekli MA, Akpinar S, Ozalay M, Ozkoc G, Cesur $\mathrm{N}$, Uysal $\mathrm{M}$, et al. Tunnel enlargement after arthroscopic anterior cruciate ligament reconstruction: comparison of bone-patellar tendonbone and hamstring autografts. Adv Ther. 2004;21(2):123-31. 
26. Sgaglione NA, Douglas JA. Allograft bone augmentation in anterior cruciate ligament reconstruction. Arthroscopy. 2004;20:171-7.

27. Zysk SP, Fraunberger P, Veihelmann A, Dörger M, Kalteis T, Maier M, et al. Tunnel enlargement and changes in synovial fluid cytokine profile following anterior cruciate ligament reconstruction with patellar tendon and hamstring tendon autografts. Knee Surg Sports Traumatol Arthrosc Off J ESSKA. 2004;12(2):98-103.

28. Hantes ME, Mastrokalos DS, Yu J, Paessler HH. The effect of early motion on tibial tunnel widening after anterior cruciate ligament replacement using hamstring tendon grafts. Arthrosc J Arthrosc Relat Surg Off Publ Arthrosc Assoc N Am Int Arthrosc Assoc. 2004;20(6):572-80.

29. Yu J, Paessler HH. Relationship between tunnel widening and different rehabilitation procedures after anterior cruciate ligament reconstruction with quadrupled hamstring tendons. Chin Med J (Engl). 2005;118(4):320-6.

30. Höher J, Möller HD, Fu FH. Bone tunnel enlargement after anterior cruciate ligament reconstruction: fact or fiction? Knee Surg Sports Traumatol Arthrosc Off J ESSKA. 1998;6(4):231-40.

31. Bhullar R, Habib A, Zhang K, de Sa D, Horner NS, Duong A, et al. Tunnel osteolysis post-ACL reconstruction: a systematic review examining select diagnostic modalities, treatment options and rehabilitation protocols. Knee Surg Sports Traumatol Arthrosc Off J ESSKA. 2019;27(2):524-33.
32. Dave LYH, Leong OK, Karim SA, Chong CH. Tunnel enlargement 5 years after anterior cruciate ligament reconstruction: a radiographic and functional evaluation. Eur J Orthop Surg Traumatol Orthop Traumatol. 2014;24(2):217-23.

33. Klein JP, Lintner DM, Downs D, Vavrenka K. The incidence and significance of femoral tunnel widening after quadrupled hamstring anterior cruciate ligament reconstruction using femoral cross pin fixation. Arthrosc J Arthrosc Relat Surg Off Publ Arthrosc Assoc N Am Int Arthrosc Assoc. 2003;19(5):470-6.

34. Jansson KA, Harilainen A, Sandelin J, Karjalainen PT, Aronen HJ, Tallroth K. Bone tunnel enlargement after anterior cruciate ligament reconstruction with the hamstring autograft and endobutton fixation techniqueA clinical, radiographic and magnetic resonance imaging study with 2 years follow-up. Knee Surg Sports Traumatol Arthrosc. 1999;7(5):290-5.

Cite this article as: Chandrashekar S, Manjunath AN, Shivakumar MS. Computed tomographic evaluation of bone tunnel enlargement following single bundle anterior cruciate ligament reconstruction with hamstring autograft. Int J Res Orthop 2022;8:81-7. 